\title{
Benign Tumors Associated With Heterozygous NTHL1 Variant
}

\author{
Danyon J. Anderson ${ }^{1}$, Andrew Boyle ${ }^{2}$, Trenton Reinicke ${ }^{3}$, Bison Woods ${ }^{1}$, Patrick Hsieh ${ }^{4}$ \\ 1. School of Medicine, Medical College of Wisconsin, Wauwatosa, USA 2. Department of Research, California Institute \\ of Technology, Pasadena, USA 3. Cancer Center, Massachusetts General Hospital Gastroenterology, Boston, USA 4. \\ Neurological Surgery, University of Southern California Keck School of Medicine, Los Angeles, USA
}

Corresponding author: Danyon J. Anderson, danderson18@cmc.edu

\begin{abstract}
NTHL1 is a tumor suppressor gene involved in base excision repair. It is associated with an increased risk for colorectal and breast cancer when two variant gene copies are inherited. However, inheriting one variant NTHL1 copy is not associated with increased tumor risk. Genetic counselors report heterozygous NTHL1 mutations as benign. We present the case of a 22-year-old patient with a heterozygous NTHL1 variant who developed an arm schwannoma, spinal schwannoma, and hepatic hemangioma. The patient also reported feeling multiple other bumps on his body but did not seek medical care due to a lack of symptoms. This case suggests that heterozygous NTHL1 variants may be implicated in tumor development.
\end{abstract}

Categories: Genetics, Oncology

Keywords: nthl1, nthl1 tumor syndrome, peripheral schwannoma, schwannoma, hemangioma, genetic testing, tumor suppressor

\section{Introduction}

NTHL1 tumor syndrome is a recessively inherited autosomal polyposis characterized by increased risk for colorectal polyposis, breast cancer, and colorectal carcinoma [1-5]. NTHL1 is a tumor suppressor gene that acts through base excision to remove and replace damaged bases from DNA, preventing mutation [6,7]. Patients homozygous for NTHL1 variants have been found to develop 14 different types of tumors affecting seven different organs [2]. NTHL1 tumor syndrome is most famously associated with increased risk for colon cancer, breast cancer, and colorectal polyposis [2]. No research has been published yet on heterozygotes at increased risk for tumor formation. In heterozygotes, one functional copy of the NTHL1 gene still facilitates proper base excision repair (BER). Regardless, there is a gap in the literature regarding the effect of being heterozygous for NTHL1 variants. Here, we present the case of an NTHL1 variant heterozygote who developed multiple benign tumors.

Review began 06/07/2021 Review ended 06/30/2021 Published 07/06/2021

(c) Copyright 2021

Anderson et al. This is an open access article distributed under the terms of the Creative Commons Attribution License CC-BY 4.0., which permits unrestricted use, distribution, and reproduction in any medium, provided the original author and source are credited.

\section{Case Presentation}

A 22-year-old white male with no significant past medical history presented to his primary care clinic two years ago for a bump on his left arm in the area of his biceps that caused intermittent pain in the distribution of the median nerve. The mass was excised and immunohistochemical staining determined it to be a benign schwannoma. A few months later, a tumor in the patient's lumbar spinal canal was found incidentally during a hematuria workup. An MRI of the lumbar spine showed a tumor (Figure 1). The tumor had not grown from a CT scan two months prior, and although the tumor could not be biopsied, it appeared to be a schwannoma or myxopapillary ependymoma. The patient reported occasional low back pain and tingling down the left foot from this tumor. No other spinal tumors were seen on a full spinal MRI. An abdominal ultrasound evaluating symptoms of acid reflux found a $1.7 \mathrm{~cm}$ hemangioma in the left hepatic lobe and pericardial effusion (Figure 2). 


\section{Cureus}

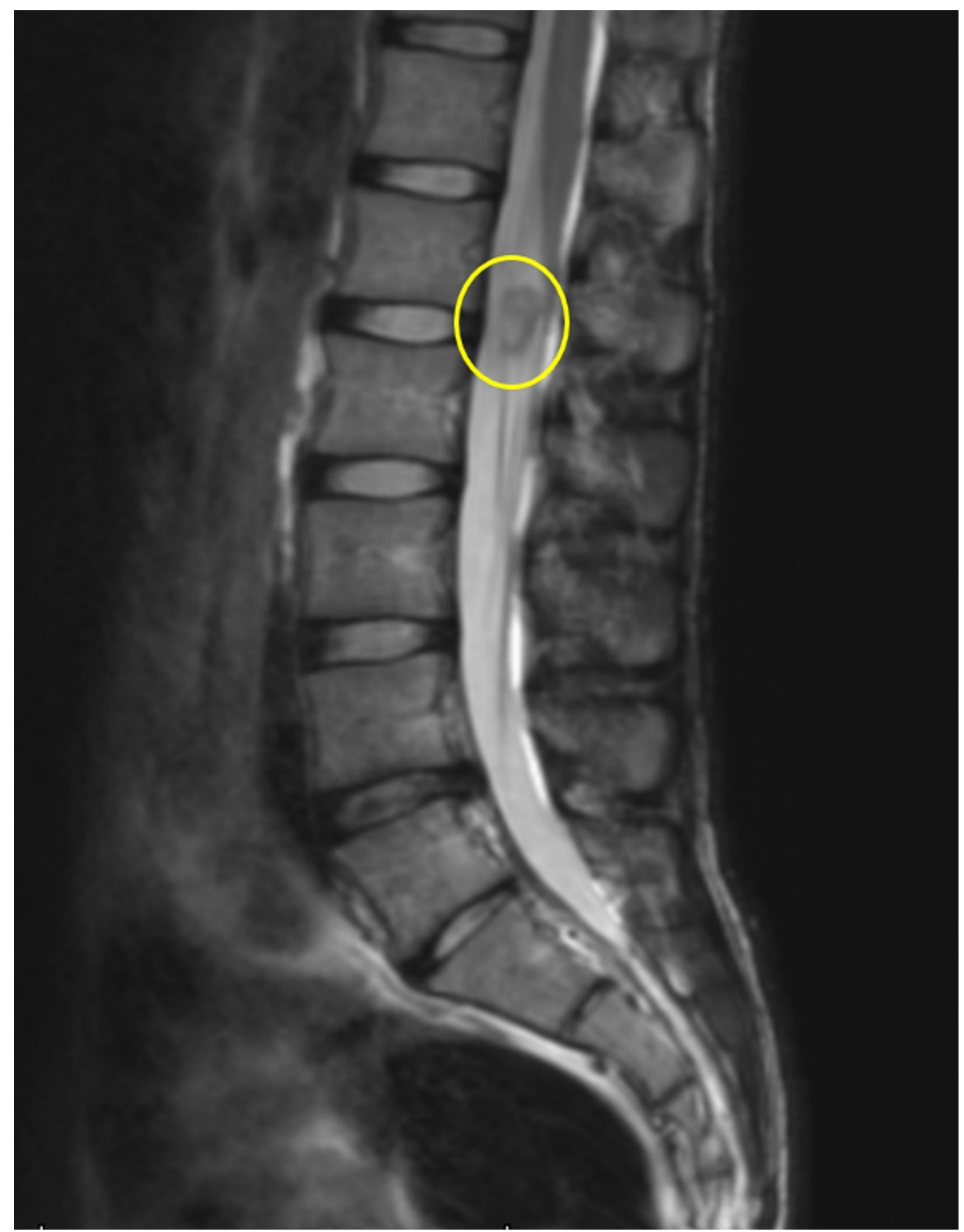

FIGURE 1: Possible schwannoma.

A redemonstration of a $1.1 \mathrm{~cm}$ intradural extramedullary lesion at the L2-3 level within the left aspect of the thecal sac lesions in the adjacent cauda equina nerve roots favored to represent a schwannoma. This does not appear appreciably changed in size or appearance over the last year. 


\section{Cureus}

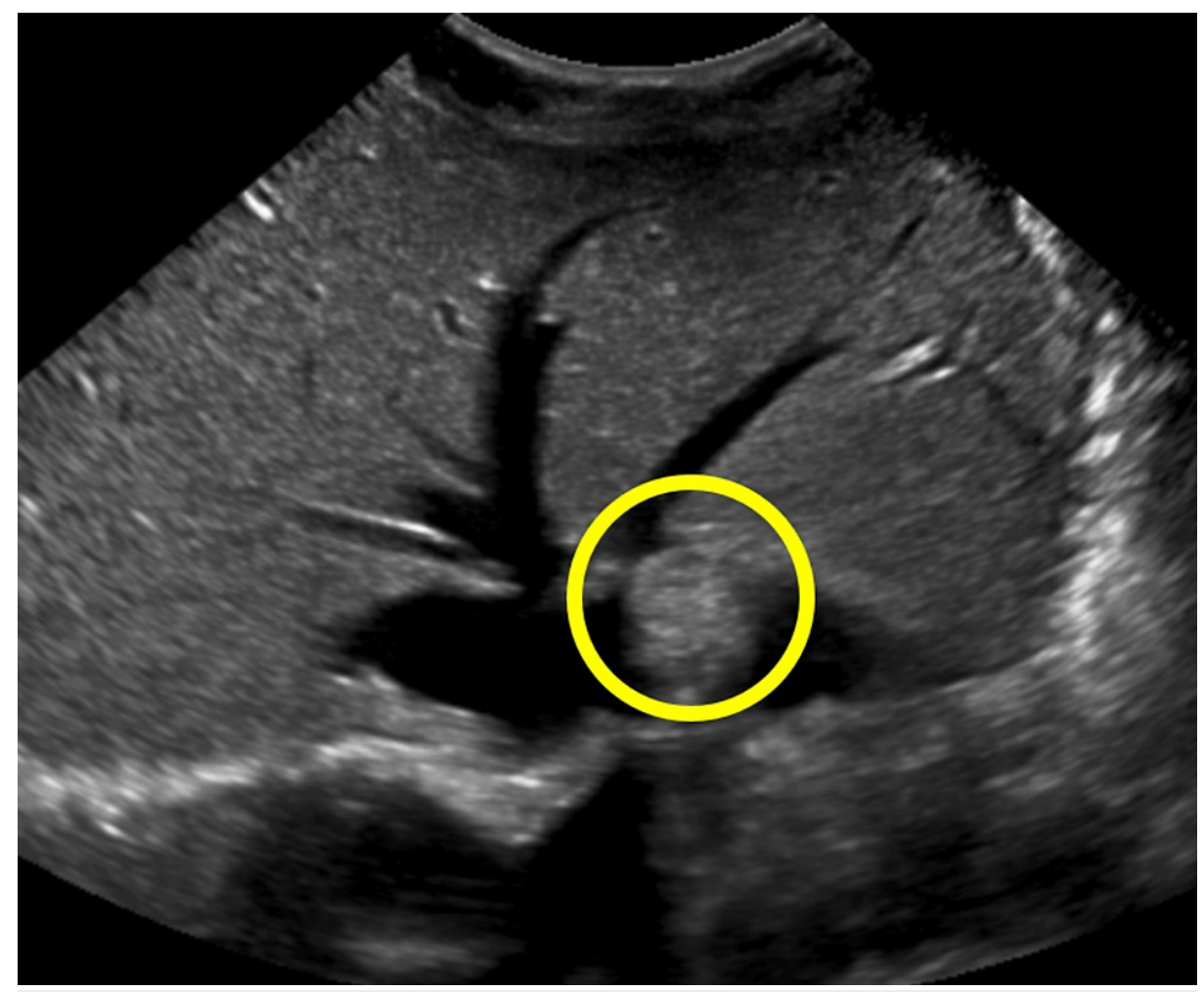

FIGURE 2: Hepatic hemangioma.

A small $1.7 \mathrm{~cm}$ echogenic focus in the peripheral aspect of the left hepatic lobe, most compatible with an incidental hemangioma.

At this point, the patient sought care to determine the cause of his tumors through genetic testing at the City of Hope. An NTHL1 heterozygous variant was found. Tests were negative for variants in 86 other genes associated with cancer or tumors. At present, the patient reports feeling more bumps on his body but has not sought care due to a lack of symptoms.

\section{Discussion}

DNA repair mechanisms are essential for maintaining efficiency and safety involved with genomic stability and proper cellular division. It is estimated that 30,000 damaged base lesions are repaired each day per cell by the BER pathway [8]. DNA glycosylases such as NTHL1, a bifunctional glycosylase, play a significant role in the BER pathway and act as the first line of defense to prevent genomic mutations caused by various environmental carcinogens such as mutagenic chemicals and distinct types of radiation [9]. Deficits in DNA damage repair pathways like BER are associated with the development of cancer, as well as a range of genetically inherited disorders, notably, NTHL1-associated polyposis (NAP) [4,8]. NAP is an autosomal recessive tumor syndrome characterized by aberrant functioning of the NTHL1 protein involved in DNA damage repair and is associated with a heightened risk for the development of colorectal cancer, breast cancer, and colorectal polyposis [1-5]. To our knowledge, the clinical diagnosis of the manifestation of multiple tumors due to having one mutant copy of NTHL1 is not described in the literature.

This patient developed a schwannoma in his upper arm, a likely schwannoma in his lumbar spinal canal, a hemangioma in his liver, a pericardial effusion, and other bumps for which he did not seek care. It is natural to suspect that there is an underlying genetic cause for his tumors. He tested negative for variants in 86 of the most common tumor-associated genes and positive for a heterozygous variant in NTHL1. It is possible that this is the first published case of a benign tumor syndrome associated with a heterozygous NTHL1 mutation. As NTHL1 is a tumor suppressor gene, inheriting one defective copy would appear to increase the risk of developing tumors through sporadic loss of function of the other copy.

If these tumors did not result from his NTHL1 mutation, it begs the question, what is the cause of these tumors if the other 86 tested genes involved in tumorigenesis were wild-type? Most nonidiopathic cases of three or more tumors in one patient are associated with a genetic cause $[10,11]$. Rare cases of multiple tumors can be linked to carcinogens such as tobacco or smoking [12-14]. However, this patient is a nonsmoker, is only 24 years old, and has not had excessive exposure to any known carcinogens. So, if his tumors are not due to his heterozygous NTHL1 variation, they are likely due to mutation in a gene that was not one of the 87 tested. This may also be a sporadic case. 
Future reports analyzing cases of multiple idiopathic tumors will be crucial in further understanding this patient's condition. More cases showing multiple idiopathic tumors in patients with heterozygous variants in NTHL1 would support our hypothesis that heterozygous NTHL1 variants may cause a syndrome of multiple benign tumors, particularly schwannomas of peripheral nerves and hemangiomas of the liver.

\section{Conclusions}

Heterozygous, in addition to homozygous, NTHL1 variants may be associated with tumor development. Our presenting patient had a heterozygous NTHL1 variant, with no other mutations in 87 tested genes, and he developed a spinal schwannoma, arm schwannoma, and hepatic hemangioma. To our knowledge, this is the first reported tumor syndrome in a patient that appears to be associated with a heterozygous variant of the NTHL1 gene. Further investigation of heterozygous NTHL1 variants will be necessary to understand its role in tumorigenesis.

\section{Additional Information \\ Disclosures}

Human subjects: Consent was obtained or waived by all participants in this study. Conflicts of interest: In compliance with the ICMJE uniform disclosure form, all authors declare the following: Payment/services info: All authors have declared that no financial support was received from any organization for the submitted work. Financial relationships: All authors have declared that they have no financial relationships at present or within the previous three years with any organizations that might have an interest in the submitted work. Other relationships: All authors have declared that there are no other relationships or activities that could appear to have influenced the submitted work.

\section{References}

1. Kuiper RP, Nielsen M, De Voer RM, Hoogerbrugge N: NTHL1 tumor syndrome. University of Washington, Seattle, Seattle, WA; 2021.

2. Grolleman JE, de Voer RM, Elsayed FA, et al.: Mutational signature analysis reveals NTHL1 deficiency to cause a multi-tumor phenotype. Cancer Cell. 2019, 11:256-66. 10.1016/j.ccell.2018.12.011

3. Elsayed FA, Grolleman JE, Ragunathan A, Buchanan DD, van Wezel T, de Voer RM: Monoallelic NTHL1 lossof-function variants and risk of polyposis and colorectal cancer. Gastroenterology. 2020, 159:2241-3. 10.1053/j.gastro.2020.08.042

4. Das L, Quintana VG, Sweasy JB: NTHL1 in genomic integrity, aging and cancer. DNA Repair. 2020, 93:102920. 10.1016/j.dnarep.2020.102920

5. Valle L, de Voer RM, Goldberg Y, et al.: Update on genetic predisposition to colorectal cancer and polyposis . Mol Aspects Med. 2019, 69:10-26. 10.1016/j.mam.2019.03.001

6. Weren RD, Ligtenberg MJ, Kets CM, et al.: A germline homozygous mutation in the base-excision repair gene NTHL1 causes adenomatous polyposis and colorectal cancer. Nat Genet. 2015, 47:668-71. 10.1038/ng.3287

7. Marsden CG, Jaruga P, Coskun E, Maher RL, Pederson DS, Dizdaroglu M, Sweasy JB: Expression of a germline variant in the N-terminal domain of the human DNA glycosylase NTHL1 induces cellular transformation without impairing enzymatic function or substrate specificity. Oncotarget. 2020, 11:2262-72. 10.18632/oncotarget.27548

8. Wallace SS, Murphy DL, Sweasy JB: Base excision repair and cancer. Cancer Lett. 2012, 327:73-89. 10.1016/j.canlet.2011.12.038

9. Lodish H, Berk A, Zipursky SL, et al.: Molecular cell biology. W. H. Freeman and Company, New York, USA; 2000. https://www.ncbi.nlm.nih.gov/books/NBK21554/.

10. Michal M, Kazakov DV, Michal M: Hybrid peripheral nerve sheath tumors: a review . Cesk Patol. 2017, 53:818.

11. Murnyák B, Szepesi R, Hortobágyi T: [Molecular genetics of familial tumour syndromes of the central nervous system]. Orv Hetil. 2015, 156:171-7. 10.1556/OH.2015.30092

12. Humble CG, Samet JM, Pathak DR, Skipper BJ: Cigarette smoking and lung cancer in 'Hispanic' whites and other whites in New Mexico. Am J Public Health. 1985, 75:145-8. 10.2105/ajph.75.2.145

13. Pande M, Amos CI, Eng C, Frazier ML: Interactions between cigarette smoking and selected polymorphisms in xenobiotic metabolizing enzymes in risk for colorectal cancer: a case-only analysis. Mol Carcinog. 2010, 49:974-80. 10.1002/mc.20682

14. Nowosielska-Grygiel J, Owczarek K, Bielińska M, Wacławek M, Olszewski J: Analysis of risk factors for oral cavity and oropharynx cancer in the authors' own material. Otolaryngol Pol. 2017, 71:23-8. 10.5604/01.3001.0009.8411 